\title{
PROBLEMS IN THE THEORY OF QUANTUM GROUPS
}

\author{
SHUZHOU WANG \\ Institut des Hautes Etudes Scientifiques \\ 35 route de Chartres, 91440-Bures-sur-Yvette, France \\ E-mail: szwang@ihes.fr \\ Current address: Department of Mathematics, University of California \\ Berkeley, CA 94720, USA \\ E-mail: szwang@math.berkeley.edu
}

\begin{abstract}
This is a collection of open problems in the theory of quantum groups. Emphasis is given to problems in the analytic aspects of the subject.

We give a collection of problems on quantum groups that are, as far as we know, still open. Many of these problems seem to be well known, though some are new. This collection is by no means meant to contain all of the important problems in the subject. However we do believe that solutions of some of these problems will give significant contribution to the theory of quantum groups. By the nature of a collection of this kind, it would be desirable to include an extensive bibliography. Because of insufficient space, we have limited ourselves to listing only those references that are cited in the problems contained in this collection. The reader can find further references in them.
\end{abstract}

1. Classifications of compact quantum groups and representations. Analysis on quantum homogeneous spaces. Recently, three natural families of compact matrix quantum groups, $A_{u}(Q), A_{o}(Q)$ and $B_{u}(Q)$, were constructed $[67,68,66,72,6,7]$. These quantum groups have remarkable properties. For instance, the $A_{u}(Q)$ 's form a universal family of compact matrix quantum groups (namely, they are the universal analogues of the ordinary unitary groups $U(n)$ ); one can take "intersections" of these quantum groups to obtain "smaller" quantum groups (in the obvious sense); the famous quantum groups $S U_{q}(2)$ are special examples of the $B_{u}(Q)$ by choosing an appropriate matrix $Q$. Unlike the quantum groups of Drinfeld-Jimbo [19, 24] and Woronowicz [81, 83],

1991 Mathematics Subject Classification: Primary 46L89, 16W30, 17B37; Secondary 22E99, 43A99, 81R50.

The paper is in final form and no version of it will be published elsewhere. 
these quantum groups cannot be obtained by the method of quantization from the classical Lie groups. To gain deeper understanding of these quantum groups and to further the theory of quantum groups, it is expected that completely new methods based more on pure mathematical considerations, other than the quantization method, should be developed. In this section, we describe some problems connected with these quantum groups.

We first recall the constructions of the compact matrix quantum groups $A_{u}(Q), A_{o}(Q)$ and $B_{u}(Q)$ (cf $\left.[67,68,66,72,6,7]\right)$. In the following, $Q$ is any invertible matrix in $G L(m, \mathbb{C}) ; u=\left(a_{i j}\right)_{i, j=1}^{m} ; u^{t}$ is the transpose of $u ; \bar{u}=\left(a_{i j}^{*}\right) ; u^{*}=\bar{u}^{t}$. The unital $C^{*}$-algebras $A_{u}(Q)$ and $A_{o}(Q)$ and $B_{u}(Q)$ are defined by generators $a_{i j}$ and the specified relations.

$A_{u}(Q): \quad u^{*} u=I_{m}=u u^{*}, \quad u^{t} Q \bar{u} Q^{-1}=I_{m}=Q \bar{u} Q^{-1} u^{t} ;$

$A_{o}(Q): \quad u^{t} Q u Q^{-1}=I_{m}=Q u Q^{-1} u^{t}, \quad \bar{u}=u$;

$B_{u}(Q): \quad u^{*} u=I_{m}=u u^{*}, \quad u^{t} Q u Q^{-1}=I_{m}=Q u Q^{-1} u^{t}$

Note that the $C^{*}$-algebra $A_{o}(Q)$ as defined above makes good sense only for positive $Q$. The quantum group $A_{o}(Q)$ here is different from the one defined in [66] with the same notation. Since the quantum group denoted by $A_{o}(Q)$ in [66] is an "intersection" of the quantum groups $A_{o}(m)$ and $B_{u}(Q)$, we believe it is better to reserve the notation $A_{o}(Q)$ for the quantum group defined above and we will do so from now on. The quantum group $B_{u}(Q)$ is also independently found by Banica, though he used our notation $A_{o}(F)$ for it $[6,7]$. If $Q=I_{m}$, then $A_{u}(Q)$ reduces to the quantum groups $A_{u}(m)$, and both $A_{o}(Q)$ and $B_{u}(Q)$ reduce to the quantum groups $A_{o}(m)$. The quantum groups $A_{u}(m)$ and $A_{o}(m)$ were constructed earlier in $[67,68]$, where a complete classification (up to isomorphism) of the quantum group structures and their underlying $C^{*}$-algebras is given. But for general $Q$, only a crude classification is obtained in [66].

Problem 1.1. Extend the method in $[67,68]$ to obtain complete classifications of the quantum groups $A_{u}(Q), A_{o}(Q)$ and $B_{u}(Q)$, as well as their underlying $C^{*}$-algebras.

In addition to the quantum groups $A_{u}(Q), A_{o}(Q)$ and $B_{u}(Q)$, there are $q$-deformations of the classical compact Lie groups (see e.g. [81, 83, 62, 61, 58]), as well as the twists of these deformations (see e.g. $[33,34,2,54,71]$ ). It is natural to ask whether there is a nice classification theory for all these quantum groups.

Problem 1.2. Develop a classification theory of simple compact quantum groups, i.e., a quantum analogue of the Killing-Cartan theory.

A satisfactory solution of the above problem would be a natural extension of the classical Killing-Cartan theory. In particular, the simple compact quantum groups would include simple compact Lie groups. A common feature of the quantum groups in $[81,83$, $62,61,58,33,34,2,54,71]$ and $[67,68,66]$ is that each of them contains a maximal torus. This could be the starting point in classification of simple compact quantum groups.

We remark that it would be impossible to classify simple quantum groups by using discrete objects, such as the Dynkin diagrams in the classification of simple Lie groups. 
We will need the continuum for such a task, as shown in [73]. In view of the classification in [73], we have the following conjecture, which is true for $K=S U(2)$ (see the end of $[73])$.

Problem 1.3. For $q, q^{\prime} \in(0,1]$, and any simple compact Lie group $K$, the quantum group $S U_{q}(2)$ is a quantum subgroup of $K_{q^{\prime}}$ (as defined in [34]) if and only if $q=q^{\prime}$.

Besides the classification of quantum groups, it is also interesting to classify their representations. In [6, 7], by decomposing tensor products of the fundamental representations, Banica classifies irreducible representations of quantum groups $A_{u}(Q)$ and $B_{u}(Q)$. In particular the irreducible representations of the quantum groups $A_{u}(m)$ and $A_{o}(m)$ are given by his scheme. In view of applications of his classification (to physics, for instance), it would be of interest to

Problem 1.4. Find interesting models for the irreducible representations classified in $[6,7]$.

It is well known that the representation theory of connected compact Lie group is described by the Cartan-Weyl theory and the Borel-Weil theory [31, 63]. Note that these theories were originally motivated from consideration of the concrete Lie groups, such as $U(m)$ and $O(m)[79,80]$. For general compact quantum groups, no such theories are available, though some interesting results in this direction are obtained in $[1,46,8]$ for the $q$-deformations of the classical Lie groups. Thus a solution of the following problem will be of great importance.

Problem 1.5. Develop analogues of the Cartan-Weyl theory and Borel-Weil theory for connected compact matrix quantum groups.

See $[67,68]$ for a definition of connected quantum groups. A solution of the above problem will no doubt enrich the theory of quantum groups substantially. It is also anticipated that, like the Cartan-Weyl theory and the Borel-Weil theory for compact Lie groups, the theories we are searching for will have diverse applications in both mathematics and physics.

In the classical Cartan-Weyl theory, the Lie algebra of a Lie group plays the central role. Likewise, in the modern theory of representation theory of semisimple Lie groups [31], the universal enveloping algebras of their Lie algebras also play dominant roles. In the theory of quantum groups developed so far, there is no good definition of a quantum Lie algebra for a compact matrix quantum group. It is expected that an appropriate definition of the quantum analogue of Lie algebras for compact matrix quantum groups will be useful for further development of the theory. In the realm of $q$-deformations, it is shown that the Drinfeld-Jimbo quantized universal enveloping algebras are duals to the compact matrix quantum groups constructed by Woronowicz et al (see [81, 83, 62, 61, 34, 56, 58]). Hence in a sense the former are the infinitesimal quantum groups of the latter. However, there are many natural compact matrix quantum groups, like $A_{u}(Q), A_{o}(Q)$ and $B_{u}(Q)$, that are not obtainable by the method of quantization. It is not clear what it should be meant by "the infinitesimal quantum groups of $A_{u}(Q), A_{o}(Q)$ and $B_{u}(Q)$ ". 
Problem 1.6. Find the right notion of infinitesimal quantum groups for compact matrix quantum groups. Determine and study the infinitesimal quantum groups of $A_{u}(Q)$, $A_{o}(Q)$ and $B_{u}(Q)$.

The quantum groups $A_{u}(Q), A_{o}(Q)$ and $B_{u}(Q)$ contain many classical compact Lie subgroups [72]. For instance the quantum groups $A_{u}(m)$ and $A_{o}(m)$ contain the groups $U(m)$ and $O(m)$ respectively (see $[67,68]$ ). Note that the construction of induced representations can be naturally extended to compact quantum groups, as well as the imprimitivity theory $[45,74]$. There is also a natural notion of quotient spaces of a compact quantum group by its quantum subgroups [47, 48, 67, 68]. The following is a summary of the problems related to these:

Problem 1.7. Understand the representations of the quantum groups $A_{u}(m)$ and $A_{o}(m)$ induced from the representations of the compact Lie groups $U(m)$ and $O(m)$. Study the quantum quotient spaces of these quantum groups. Develop the theory of Gelfand pairs for compact quantum groups and find the applications thereof. Gain a better understanding of the imprimitivity theory for compact quantum groups in the light of Gelfand pairs and quantum quotient spaces.

Let $E / F$ be a quadratic extension of a field $F(\operatorname{char}(F)=0)$. Let $\sigma$ be the generator of the group $\operatorname{Gal}(E / F)$. For any $Q$ in $G L(n, E)$, we can still define the Hopf *-algebras $A_{u}(Q), A_{o}(Q)$ and $B_{u}(Q)$ as above, where the conjugation of the matrix $Q$ is defined by using $\sigma$.

Problem 1.8. Understand the Hopf *algebras $A_{u}(Q), A_{o}(Q)$ and $B_{u}(Q)$. Is there any connection between these Hopf *algebras and the theory of automorphic representations [10, 25]?

2. Problems on finite quantum groups. Though the $q$-deformations of compact Lie groups is well understood, finite quantum groups are still poorly studied. Both as objects of great mathematical interest, like finite groups, and as objects with potential important applications in theoretical physics $[14,16]$, finite quantum groups worth devoted efforts of study.

To start with, we should have a rich supply of natural and interesting examples, which is still lacking so far, though a few non-trivial examples have been studied $[30,5]$. In the theory of finite groups, the finite groups of Lie type (Chevalley groups) are one of the most important classes of finite groups. In view of the fact that classical Lie groups have $q$-deformations, a natural question in this connection is

Problem 2.1. Do finite groups of Lie type have an analogue of q-deformations into finite quantum groups?

In [75], a deformation of Rieffel type for finite groups (actually finite quantum groups) that contain an abelian subgroup is constructed. However this deformation is not the analogue of the $q$-deformations, it is rather an analogue of the Drinfeld twisting (see $[33,34,54,55,71])$. We briefly recall this construction. Let $(A, \Delta)$ be a finite quantum group (in the sense that $A=C(G), \Delta$ being the coproduct on $A$ ) with the property that 
its maximal subgroup $X(A)=\left\{{ }^{*}\right.$-homomorphisms from $A$ into $\left.\mathbb{C}\right\}$ contains an abelian subgroup $T$. Any such group $T$ has the form

$$
T \cong \mathbb{Z} / n_{1} \mathbb{Z} \oplus \mathbb{Z} / n_{2} \mathbb{Z} \oplus \cdots \oplus \mathbb{Z} / n_{l} \mathbb{Z},
$$

for some positive integers $n_{1}, n_{2}, \cdots, n_{l}$. Define an action $\alpha$ of $H:=T \times T$ on the $C^{*}$ algebra $A$ as follows:

$$
\alpha_{(s, u)}=\lambda_{s} \rho_{u}
$$

where

$$
\lambda_{s}=\left(E_{(-s)} \otimes i d\right) \Delta, \quad \rho_{u}=\left(i d \otimes E_{u}\right) \Delta,
$$

$i d$ being the identity map on $A$ and $E_{u}$ the evaluation functional on $C(T)$ corresponding to $u$. Fix $l$ generators for $T$. Every homomorphism $S$ on $T$ is uniquely determined by its action on these generators and can be represented by an $l \times l$ matrix $\left(s_{i j}\right)$ with integer coefficients $s_{i j}$ (viewed as homomorphisms from $\mathbb{Z} / n_{j} \mathbb{Z}$ to $\mathbb{Z} / n_{i} \mathbb{Z}$ ). Let $S$ be a homomorphism of $T$ whose matrix representation in the above sense is skew-symmetric. Note that the skew-symmetricity of the matrix for $S$ is independent of the choice of the generators of $T$. Let $J$ be the homomorphism $S \oplus(-S)$ on $H$. Define a new product $\times_{J}$ on $A$ by

$$
a \times_{J} b=\sum_{s, t, u, v \in T} \alpha_{J(s, u)}(a) \alpha_{(t, v)}(b)<s, t><u, v>, \quad a, b \in A,
$$

where $\langle s, t\rangle$ is the bilinear form on $T$ (with values in the circle group) that identifies $T$ with its dual group $\hat{T}$. The main result of [75] is: Under the same coproduct $\Delta$ of $A$, the deformation $\left(A, \times_{J}\right)$ is still a finite quantum group containing $T$ as a subgroup. In particular, a finite group that contains a finite abelian group of order of non-prime power can be deformed into a finite quantum group. This construction is related to the ones in $[34,54,55,71,32,23]$. Just as the quantum groups obtained by the Rieffel quantization $[54,55,71]$, the quantum groups obtained in this manner contain the original finite abelian group $T$ and can be deformed again. However, unlike the construction in [32, 23], which imposes cocyle type conditions in addition to the existence of an abelian subgroup, our construction above is in some sense canonically associated with the abelian subgroup. Because of this canonical feature of the construction, we believe more interesting secrets associated with this construction need to be revealed. To gain better understanding of this construction, it is desirable to solve the following

Problem 2.2. Characterize the finite quantum groups that can be obtained as deformations of finite groups in the manner above. Find their isomorphic invariant(s).

For a number field $F$, the Galois group $\operatorname{Gal}(\bar{F} / F)$ is a compact group having a big abelian quotient group $\operatorname{Gal}(\bar{F} / F)^{a b}, \bar{F}$ being the algebraic closure of $F$. The group $\operatorname{Gal}(\bar{F} / F)^{a b}$ is well-understood (class field theory). But the nonabelian part of the group $\operatorname{Gal}(\bar{F} / F)$ is still an object of immense interest in current research in number theory and representation theory. It would be interesting to understand what light the deformations of $\operatorname{Gal}(E / F)$ in the above sense will shed on $\operatorname{Gal}(\bar{F} / F)$, where $E$ is a finite extension of $F$. 
Problem 2.3. Investigate the deformations of $G a l(E / F)$ in detail and find their applications in number theory.

In view of the construction of $A_{u}(Q), A_{o}(Q)$ and $B_{u}(Q)$ and their properties, we are naturally led to the following

Problem 2.4. Find interesting finite quantum subgroups of $A_{u}(Q), A_{o}(Q)$ and $B_{u}(Q)$ by "taking their intersections or quotients".

In view of application in theoretical physics $[14,16]$, it is important to solve the following problem.

Problem 2.5. (Due to Connes) Find finite quantum symmetry groups of a finite quantum space (i.e. a finite dimensional algebra).

A good understanding of enough examples of finite quantum groups should make it plausible to solve the following problems, which have remained open for a long period of time:

Problem 2.6. Does the dimension of an irreducible representation of a finite quantum group divide the order of the finite quantum group (cf [28])?

PROBLEM 2.7. Is the classes of non-isomorphic finite quantum groups of a given order finite?

Other problems in finite quantum groups naturally arise [17, 18].

3. Problems on non-compact quantum groups. Non-compact quantum groups seem to present the most tantalizing problems. Though a few concrete non-compact quantum groups have been studied in detail (see e.g. [29, 53, 64, 39, 40, 49, 50, 85, 3, 4]), there is not a satisfactory analytic theory for complex semisimple quantum groups $[19,24]$. Note however that the algebraic aspects of complex semisimple quantum groups are quite well understood (see e.g. $[57,36,35,13]$ ). It is quite easy to see that parts of the work of Podles, Pusz and Woronowicz on the quantum Lorentz group [49, 50, 51] can be generalized to arbitrary complex quantum groups. It is expected that the algebraic aspects of these quantum groups will serve as main tools for understanding their analytic aspects. Namely, we can expect an analytic theory for all complex semisimple quantum groups that is in line with the tradition of Gelfand and Naimark and Harish-Chandra for complex semisimple Lie groups.

Problem 3.1. Study the unitary representations of complex semisimple quantum groups by extending the works in [39, 40, 49, 50, 51].

PROBlem 3.2. Develop a theory of admissible representations for complex semisimple quantum groups [31].

Now we turn to the abstract theory of locally compact quantum groups. There is still no satisfactory notion of a locally compact quantum group, though some recent attempts are made $[5,4,60,41,43,88]$. The main problem is

PROBLem 3.3. Find a notion of locally compact quantum groups that contains ring groups (i.e. Kac algebras) [22], compact quantum groups [82, 86, 67, 68], and all the 
interesting non-compact quantum groups constructed so far (e.g. [29, 53, 55, 64, 49, 85]), and entails the existence and uniqueness of the Haar measure.

In practice, the most useful Lie groups are matrix groups (linear groups). In view of this, it is more practical to solve the following problem first:

Problem 3.4. Develop a theory of non-compact matrix quantum groups along the lines of [82].

We remark that some tools needed to solve the problem above have been developed by Woronowicz [85, 88].

The most tractable classes of general locally compact quantum groups seem to be the regular ones, i.e., those corresponding to the regular (or semi-regular, or manageable) multiplicative unitaries $[5,4,88]$. For the quantum groups $S_{V}$ and $\hat{S}_{V}$ associated with such a multiplicative unitary $V$ (see [5] for the meaning of these symbols and related definitions), the existence and uniqueness of the Haar measures should be determinable by the following procedure. Because of the regularity (or semi-regularity, or manageability) of $V$, one should be able to construct two left Hilbert algebras $H_{V}$ and $\hat{H}_{V}$ sitting in $S_{V}$ and $\hat{S}_{V}$ respectively, in exactly the same way as the construction of the left Hilbert algebras $H_{V}=C_{c}(G)$ and $\hat{H}_{V}=C_{c}(G)$ sitting in $S_{V}=C_{\infty}(G)$ and $\hat{S}_{V}=C_{r}^{*}(G)$ respectively when $V$ is the multiplicative unitary associated with a locally compact group $G$. (One can also construct two right Hilbert algebras.) The unique modular weights associated with the left Hilbert algebras $H_{V}$ and $\hat{H}_{V}$ should be the Haar measures for the quantum groups $S_{V}$ and $\hat{S}_{V}$. For compact quantum groups and the quantum groups associated with Kac algebras, the idea above for the Haar measure can be easily verified. The following remains to be settled.

Problem 3.5. For any regular (or semi-regular or manageable) multiplicative unitary $V$, give a rigorous construction of left Hilbert algebras $H_{V}$ and $\hat{H}_{V}$ suggested above, and show that the unique modular weights associated with them are left Haar measures on $S_{V}$ and $\hat{S}_{V}$ respectively (that is, they are invariant under left translations).

Similarly, one may formulate a conjecture for right Haar measures.

Experience shows that once the Haar measures are found, it is not hard to to verify the following conjecture of Skandalis (see the end of [60]).

Problem 3.6. Let $\phi$ and $\psi$ (resp. $\hat{\phi}$ and $\hat{\psi}$ ) be the left and right Haar measures on $S_{V}\left(\right.$ resp. $\left.\hat{S}_{V}\right)$. There should exist positive unbounded operators $F$ and $\hat{F}$ affiliated with the the centralizers of $\phi$ and $\hat{\phi}$ such that for all $x \in S_{V}$ and $y \in \hat{S}_{V}$, one has

$$
\psi(x)=\phi(F x F), \quad \hat{\psi}(y)=\hat{\phi}(\hat{F} y \hat{F}) .
$$

Let $\Delta_{\phi}, \Delta_{\psi}, \Delta_{\hat{\phi}}, \Delta_{\hat{\psi}}$ be the modular operator associated with the weights $\phi, \psi, \hat{\phi}, \hat{\psi}$, respectively. Then one has

$$
\begin{gathered}
\Delta_{\phi}=L\left(F^{-1}\right) R\left(F^{-1}\right) \lambda(\hat{F}) \rho\left(\hat{F}^{-1}\right), \quad \Delta_{\psi}=L(F) R(F) \lambda(\hat{F}) \rho\left(\hat{F}^{-1}\right), \\
\Delta_{\hat{\phi}}=L\left(F^{-1}\right) R(F) \lambda\left(\hat{F}^{-1}\right) \rho\left(\hat{F}^{-1}\right), \quad \Delta_{\hat{\psi}}=L\left(F^{-1}\right) R(F) \lambda\left(\hat{F}^{-1}\right) \rho\left(\hat{F}^{-1}\right),
\end{gathered}
$$

where $L, R, \lambda, \rho$ are defined as in [5]. 


\section{Other problems. Noncommutative geometry with quantum groups}

Problem 4.1. (cf [81, 84]) Develop a canonical differential calculus on a compact matrix quantum group $G=(A, u)$ by introducing a canonical spectral triple $(\mathcal{A}, H, D)$ (in the sense of Connes [14, 16]) so that the Haar integral $h$ is related to the quantized integral $\operatorname{Tr}_{\omega}$ of Connes by the following formula

$$
h(a)=\operatorname{Tr}_{\omega}\left(a|D|^{-d}\right), \quad a \in \mathcal{A} .
$$

Problem 4.2. (Due to P.M. Hajac) Find the structure quantum groups of the quantum vetor bundles for $T_{\theta}^{2}$ [52]. One should be able to use the universal quantum groups [66] to find clues to these quantum groups (by reduction of the structure group).

Find interesting examples of quantum principal bundles [11, 27].

Problem 4.3. From the philosophy of the Tannaka-Krein duality [83, 70], one can recover a compact quantum group $G$ from its dual $\hat{G}$. Calculate the cyclic theory of the Krein algebra $\mathcal{A}(G)$ (see [70]) of a compact quantum group in terms of $\hat{G}$. For the special cases of compact Lie groups, groups algebras of discrete groups, and the quantum $S U(2)$ groups, this is done respectively in [15, 12, 42].

\section{Structure of free product of quantum groups}

Problem 4.4. Compact quantum groups are the same as discrete quantum groups by duality (see [49, 5, 70, 67, 21, 65]). In view of the free product construction of quantum groups [67, 68], it is interesting to know if there are analogs of the theorems of Gruško, Kurosh and Schreier (see Serre [59]).

PROBLEM 4.5. Many constructions of quantum groups (Hopf algebras) can be viewed as constructions of Woronowicz Hopf $C^{*}$-ideals in the free product $A * B$ of two quantum groups (see [37, 69]). Develop a classification theory for such constructions (or equivalently, ideals).

\section{Quantum groups and operator algebras}

Problem 4.6. There are few examples of actions of quantum groups on operator algebras (see [44, 76]), though there is a general theory for such actions [9]. It is very interesting to decide if the quantum groups $S U_{q}(2)$ (with $q \in[-1,1] \backslash\{0\}$ ) admitts ergodic actions on the hyperfinite $I I_{1}$ factor $R$, though the group $S U(2)$ admitts no such actions [78]. The root of unity case $q=-1$ should be exceptionally interesting.

Problem 4.7. From the fundamental representation of the universal compact quantum group $A_{u}(m)[68,66]$, one can construct a natural action of $A_{u}(m)$ on the hyperfinite $I I_{1}$ factor $R$ [76]. This action extends the natural action of the unitary group $U(m)$ (note that $U(m)$ is a subgroup of $A_{u}(m)$ [68]) on $R$, and by restriction, it gives rise to a natural action of its quantum subgroup $S U_{-1}(m)$ on $R$. In view of 4.7 of [26], it would be interesting to relate the subfactors of $R$ and (finite) quantum subgroups of $A_{u}(m)$ and $\mathrm{SU}_{-1}(\mathrm{~m})$ from this action.

Problem 4.8. Study the von Neumann algebras generated by the GNS representations of the Haar measures of the quantum groups $A_{u}(n)$. Try to relate these von Neumann 
algebras to the free group $I I_{1}$ factors $L\left(F_{n}\right)$ and to von Neumann's famous problem on these factors (See Chapter $V$ of [14]).

Acknowlegdement. The author gratefully acknowledges the financial support of the IHES during the period when this work was done. He would also like to thank the Director Professor J.-P. Bourguignon and the staff of the IHES for their hospitality and for creating an excellent scientific atmoshpere.

Note on Problem 2.5: In our paper Quantum symmetry groups of finite spaces under preparation, we have found the quantum automorphism groups of finite spaces that have no additional (noncommutative) Riemmanian structure. These quantum groups are generically infinite. All finite quantum symmetry groups of such spaces are quantum subgroups of the quantum groups in that paper.

\section{References}

[1] H.H. Andersen, P. Polo and K. Wen, Representations of quantum algebras, Invent. Math. 104 (1991), 1-59.

[2] N. Andruskiewitsch and B. Enriquez, Examples of compact matrix pseudogroups arising from twisting operation, Commun. Math. Phys. 149 (1992), 195-208.

[3] S. Baaj, Représentation régulière du groupe quantique $E_{\mu}(2)$ de Woronowicz, $C$. $R$. Acad. Sci. Paris t. 314, Serie I (1992), 1021-1026.

[4] S. Baaj, Représentation régulière du groupe quantique des déplacements de Woronowicz, in Recent Advances in Operator Algebras, Astérisque 232 (1995), 11-48.

[5] S. Baaj and G. Skandalis, Unitaires multiplicatifs et dualité pour les produits croisés de $C^{*}$-algèbres, Ann. Sci. Ec. Norm. Sup. 26 (1993), 425-488.

[6] T. Banica, Théorie des représentations du groupe quantique compact libre $\mathrm{O}(\mathrm{n}), C . R$. Acad. Sci. Paris t. 322, Serie I (1996), 241-244.

[7] T. Banica, Le groupe quantique compact libre U(n), Preprint, Unversity of Paris VII, 1996.

[8] L. C. Biedenharn and M. A. Lohe, An extension of the Borel-Weil construction to the quantum group $U_{q}(n)$, Comm. Math. Phys. 146 (1992), 483-504.

[9] F. Boca, Ergodic actions of compact matrix pseudogroups on $C^{*}$-algebras, in Recent $A d$ vances in Operator Algebras, Astérisque 232 (1995), 93-109.

[10] A. Borel et al (ed): Automorphic Forms, Representations, and L-Functions, Proc. Symp. Pure Math., Vol 33, Amer. Math. Soc., 1979.

[11] T. Brzeziński and S. Majid, Quantum group gauge theory on quantum spaces, Commun. Math. Phys. 157 (1993), 591-638.

[12] D. Burghelea, The cyclic homology of the groups rings, Comment. Math. Helv. 60 (1985), 354-365.

[13] V. Chari and A. Pressley, A Guide to Quantum Groups, Cambridge University Press, 1994.

[14] A. Connes, Noncommutative Geometry, Academic Press, 1994.

[15] A. Connes, Non-commutative differential geometry, Publ. Math. IHES 62 (1985), 41-144.

[16] A. Connes, Noncommutative geometry and reality, J. Math. Phys. 36 No 11 (1996).

[17] C. W. Curtis and I. Reiner, Methods in Representation Theory, I, Wiley, 1981.

[18] C. W. Curtis and I. Reiner, Methods in Representation Theory, II, Wiley, 1987. 
[19] V. G. Drinfeld, Quantum groups, Proc. ICM-1986, Berkeley, Vol I, Amer. Math. Soc., Providence, R.I., 1987, pp798-820.

[20] M. Dubois-Violette and G. Launer, The quantum group of a non-degenerate bilinear form, Phys. Lett. B 245 (1990), 175-177.

[21] E. Effros and Z.-J. Ruan, Discrete quantum groups, I. The Haar measure, Internat. J. Math. 5 (1994), 681-723.

[22] M. Enoch and J. M. Schwartz, Kac Algebras and Duality of Locally Compact Groups, Springer-Verlag, New York, 1992.

[23] M. Enock and L. Vainerman, Deformation of a Kac algebra by an abelian subgroup, Preprint, Univ. Pierre et Marrie Curie, Aug, 1995.

[24] L. D. Faddeev, N. Y. Reshetikhin and L. A. Takhtajan, Quantization of Lie groups and Lie algebras, Algebra and Analysis 1 (1990), 193-225.

[25] S. S. Gelbart, Automorphic Forms on Adele Groups, Annals of Mathematics Studies 83, Princeton University Press, 1975.

[26] F. M. Goodman, P. de la Harpe and V. F. R. Jones, Coxeter Graphs and Towers of Algebras, MSRI Publ. 14, Springer-Verlag, 1989.

[27] P. M. Hajac, Strong Connections and $U_{q}(2)-$ Yang-Mills Theory on Quantum Principal Bundles, Ph.D Thesis, University of California at Berkeley, May, 1994.

[28] G. Kac, Certain arithmetic properties of ring groups, Funct. Anal. Appl. 6 (1972), 158-160.

[29] G. Kac and V. Palyutkin, An example of a ring group generated by Lie groups, Ukrain. Math. J. 16 (1964), 99-105.

[30] G. Kac and V. Palyutkin, Finite ring groups, Trans. Moscow Math. Soc. 15 (1966), 251-294.

[31] A. W. Knapp, Representation Theory of Semisimple Groups: An Overview Based on Examples, Princeton University Press, 1986.

[32] M. B. Landstad, Quantizations arising from abelian subgroups, Internat. J. Math. 5 (1994), 897-936.

[33] S. Levendorskii, Twisted algebra of functions on compact quantum group and their representations, St. Petersburg Math. J. 3:2 (1992), 405-423.

[34] S. Levendorskii and Y. Soibelman, Algebra of functions on compact quantum groups, Schubert cells, and quantum tori, Comm. Math. Phys. 139, (1991), 141-170.

[35] G. Lusztig, Introduction to Quantum Groups, Progress in Mathematics Vol 110, Birkhauser, 1993.

[36] G. Lusztig, Quantum deformations of certain simple modules over enveloping algebras, Adv. in Math. 70 (1988), 237-249.

[37] S. Majid, Physics for algebraists: non-commutative and noncocommutative Hopf algebras by a bycrossproduct construction, J. Algebra 130 (1990), 17-64.

[38] Y. Manin, Quantum Groups and Noncommutative Geometry, Publications du C.R.M. 1561, Univ de Montreal, 1988.

[39] T. Masuda, K. Mimachi, Y. Nakagami, M. Noumi, Y. Saburi and K. Ueno, Unitary representations of the quantum group $S U_{q}(1,1)$ : I-Structure of the dual space of $U_{q} \mathfrak{s l}(2)$, Lett. Math. Phys. 19 (1990), 187-194.

[40] T. Masuda, K. Mimachi, Y. Nakagami, M. Noumi, Y. Saburi and K. Ueno, Unitary representations of the quantum group $S U_{q}(1,1)$ : II-Matrix elements of unitary representations and basic hypergeometric functions, Lett. Math. Phys. 19 (1990), 195-204.

[41] T. Masuda and Y. Nakagami, A von Neumann algebra framework for the duality of the quantum groups, Publ. RIMS 30:5 (1994), 799-850. 
[42] T. Masuda, Y. Nakagami and J. Watanabe, Noncommutative geometry on the quantum $S U(2)$ I: An algebraic viewpoint, K-theory 4 (1990), 157-180.

[43] T. Masuda, Y. Nakagami and S. L. Woronowicz, A $C^{*}$-algebraic framework for quantum groups, to appear.

[44] Y. Nakagami, Takesaki duality for the crossed product by quantum groups, in Quantum and Non-Commutative Analysis, H. Araki ed., Kluwer Academic Publishers, 1993, 263-281.

[45] A. Pal, Induced representation and Frobenius reciprocity for compact quantum groups, Proc. Indian Acad. Sci. (Math. Sci.) 105:2 (1995), 157-167.

[46] B. Parshall and J. Wang, Quantum linear groups, Memoirs AMS 439, 1991.

[47] P. Podleś, Quantum spheres, Letters Math. Phys. 14 (1987), 193-202.

[48] P. Podleś, Symmetries of quantum spaces. Subgroups and quotient spaces of quantum $S U(2)$ and $S O(3)$ groups, Comm. Math. Phys. 170 (1995), 1-20.

[49] P. Podleś and S. L. Woronowicz, Quantum deformation of Lorentz group, Comm. Math. Phys. 130 (1990), 381-431.

[50] W. Pusz, Irreducible unitary representations of quantum Lorentz group, Comm. Math. Phys. 152 (1993), 591-626.

[51] W. Pusz and S. L. Woronowicz, Unitary representations of quantum Lorentz group, Preprint, Warsaw University, 1993.

[52] M. Rieffel, Noncommutative tori, Contemp. Math 105 (1990), 191-211.

[53] M. Rieffel, Some solvable quantum groups, in Operator Algebras and Topology, W. B. Arveson, A. S. Mischenko, M. Putinar, M. A. Rieffel and S. Stratila, ed., Proc. Conf. Operator Algebras and their Connection with Topology and Ergodic Theory, 2nd Conference, Romania, 1989, Pitman Research Notes in Mathematics 270, Longman, Burnt Mill, England, 1992, pp146-159.

[54] M. Rieffel, Compact quantum groups associated with toral subgroups, Contemp. Math. 145 (1993), 465-491.

[55] M. Rieffel, Non-compact quantum groups associated with abelian subgroups, Comm. Math. Phys. 171 (1995), 181-201.

[56] M. Rosso, Comparaison des groupes $S U(2)$ quantiques de Drinfeld et Woronowicz, C. $R$. Acad. Sci. Paris 304 (1987), 323-326.

[57] M. Rosso, Finite dimensional representations of the quantum analog of the enveloping algebra of a complex semisimple Lie algebra, Comm. Math. Phys. 117 (1988), 581-593.

[58] M. Rosso, Algèbres enveloppantes quantifiées, groupes quantiques compacts de matrices et calcul différentiel non-commutatif, Duke Math. J. 61 (1990), 11-40.

[59] J.-P. Serre, Arbres, amalgames, $S L_{2}$, Soc. Math. France, Asterisque 46 (1977).

[60] G. Skandalis, Operator algebras and duality, Proc. ICM-1990, Kyoto, Vol II, Springer, New York, 1991, pp997-1009.

[61] Y. Soibelman, Algebra of functions on a compact quantum group and its representations, Leningrad Math. J. 2:1 (1990), 161-178.

[62] L. Vaksman and Y. Soibelman, The algebra of functions on quantum SU(2), Funct. Anal. ego Pril. 223, (1988), 1-14.

[63] V. S. Varadarajan, Lie Groups, Lie Algebras, and their Representations, Graduate Text in Math, no. 102, Springer, 1984.

[64] A. Van Daele, Quantum deformation of the Heisenberg group, Proc. Satellite Conf. of ICM-90, Current Topics in Operator Algebras, 314-325.

[65] A. Van Daele, Discrete quantum groups, J. Alg. 180 (1996), 431-444. 
[66] A. Van Daele and S. Z. Wang, Universal quantum groups, International J. Math 7:2 (1996), 255-264.

[67] S. Z. Wang, General Constructions of Compact Quantum Groups, Ph.D Thesis, University of California at Berkeley, March, 1993.

[68] S. Z. Wang, Free products of compact quantum groups, Comm. Math. Phys. 167 (1995), 671-692.

[69] S. Z. Wang, Tensor products and crossed products of compact quantum groups, Proc. London Math. Soc. 71 (1995), 695-720.

[70] S. Z. Wang, Krein duality for compact quantum groups, J. Math. Phys. 38 No. 1 (1997).

[71] S. Z. Wang, Deformations of compact quantum groups via Rieffel's quantization, Commun. Math. Phys. 178 (1996), 747-764.

[72] S. Z. Wang, New classes of compact quantum groups, Lecture notes for talks at the University of Amsterdam and the University of Warsaw, January and March, 1995.

[73] S. Z. Wang, Classification of quantum groups $S U_{q}(n)$, to appear in J. London Math. Soc.

[74] S. Z. Wang, Imprimitivity theory for compact quantum groups, In preparation.

[75] S. Z. Wang, Rieffel type discrete deformations of finite quantum groups, In preparation.

[76] S. Z. Wang, Actions of universal quantum groups on operator algebras, In preparation.

[77] S. Z. Wang, Quantum symmetry groups of finite spaces, In preparation.

[78] A. Wassermann, Ergodic actions of compact groups on operator algebras III: Classification for $S U(2)$, Invent. Math. 93 (1988), 309-355.

[79] H. Weyl, The Classical Groups, Princeton University Press, 1946.

[80] H. Weyl, Theory of Groups and Quantum Mechanics, Princeton University Press, 1930.

[81] S. L. Woronowicz, Twisted $S U(2)$ group. An example of noncommutative differential calculus, Publ. RIMS, Kyoto Univ. 23, (1987), 117-181.

[82] S. L. Woronowicz, Compact matrix pseudogroups, Comm. Math. Phys. 111, (1987), 613-665.

[83] S. L. Woronowicz, Tannaka-Krein duality for compact matrix pseudogroups. Twisted $S U(N)$ groups, Invent. Math. 93 (1988), 35-76.

[84] S. L. Woronowicz, Differential calculus on compact matrix pseudogroups (quantum groups), Comm. Math. Phys. 122 (1989), 125-170.

[85] S. L. Woronowicz, Unbounded elements affiliated with $C^{*}$-algebras and non-compact quantum groups, Comm. Math. Phys. 136 (1991), 399-432.

[86] S. L. Woronowicz, Compact quantum groups, Preprint, Warsaw University, 1992

[87] S. L. Woronowicz, $C^{*}$-algebras generated by unbounded elements, Reviews in Mathematical Physics 7:3 (1995), 481-521.

[88] S. L. Woronowicz, From multiplicative unitaries to quantum groups, Preprint, Warsaw University, March, 1995. 